\title{
A Chopper Stabilization Audio Instrumentation Amplifier for IoT Applications
}

\author{
Jamel Nebhen ${ }^{1, *(\mathbb{D})}$, Pietro M. Ferreira ${ }^{2,3}$ a and Sofiene Mansouri ${ }^{1}$ \\ 1 College of Computer Engineering and Sciences, Prince Sattam bin Abdulaziz University, P.O. Box 151, \\ Alkharj 11942, Saudi Arabia; s.mansouri@psau.edu.sa \\ 2 Lab. de Génie Electrique et Electronique de Paris, Paris-Saclay, CentraleSupélec, CNRS, 91192 Gif-sur-Yvette, \\ France; maris@ieee.org \\ 3 Lab. de Génie Electrique et Electronique de Paris, Sorbonne Université, CNRS, 75252 Paris, France \\ * Correspondence: j.nebhen@psau.edu.sa
}

Received: 23 March 2020; Accepted: 7 April 2020; Published: 16 April 2020

\begin{abstract}
A low-noise instrumentation amplifier dedicated to a nano- and micro-electro-mechanical system (M\&NEMS) microphone for the use in Internet of Things (IoT) applications is presented. The piezoresistive sensor and the electronic interface are respectively, silicon nanowires and an instrumentation amplifier. To design an instrumentation amplifier for IoT applications, different trade-offs are discussed like power consumption, gain, noise and sensitivity. Because the most critical noisy block is the amplifier, a delay-time chopper stabilization (CHS) technique is implemented around it to eliminate its offset and $1 / f$ noise. The low-noise instrumentation amplifier is implemented in a 65-nm CMOS (Complementary metal-oxide-semiconductor) technology. The supply voltage is $2.5 \mathrm{~V}$ while the power consumption is $0.4 \mathrm{~mW}$ and the core area is $1 \mathrm{~mm}^{2}$. The circuit of the M\&NEMS microphone and the amplifier was fabricated and measured. From measurement results over a signal bandwidth of $20 \mathrm{kHz}$, it achieves a signal-to-noise ratio (SNR) of $77 \mathrm{~dB}$.
\end{abstract}

Keywords: M\&NEM microphone; instrumentation amplifier; silicon nanowire; low-noise; IoT; chopper stabilization

\section{Introduction}

The Internet of Things (IoT) is now recognized by industry, and in particular the electronics industry, as one of the main engines of growth for the decade to come, if not more. The IoT refers to any application taking advantage of the networking of objects capable of interacting with their environment to measure key parameters of this environment, then to transmit this data for analysis, sometimes in real time, and decision making to control or optimize a system. Detection is the starting point for the IoT and smart home applications. It is also the first problem faced by followers and professional designers. The design of many economical transducers such as accelerometers, force sensors, extensometers and pressure transducers is based on resistive Wheatstone bridges for differential voltages in millivolts $(\mathrm{mV})$. Before going into detail, it is essential to accurately capture these low-level signals and amplify them to levels compatible with analog-to-digital converters (ADCs) without direct current (DC) offset or noise. Likewise, current detection using high potential ammeter shunts requires amplifiers without inputs referenced to ground and capable of tolerating high common mode voltages. Micro-electro-mechanical systems (MEMS) are sensors or actuators whose lateral dimensions and thickness are of the order of a micrometer. For decades until today, MEMS sensors have been manufactured on a large scale for many consumer applications such as aerospace [1], inertial sensors in mobile phones such as gyrometers and accelerometers [2,3], video game controllers and airbag triggers. These devices, which are the basis of 
research tools [4], have reached a sufficient maturity to be directly developed and integrated by large industrial groups such as STMicroelectronics [5].

In the world of transistors, it is known that the reduction of dimensions mainly allows integrating more devices on a given surface. Therefore, it enables to reduce the manufacturing cost of the transistor, to increase the performance of the integrated circuit, and reduce the operating voltage. With regard to sensors, reduced dimensions are also other benefits that enabled the development of emerging applications. Therefore, since the 2000s, these sensors have been reduced to the nanometer scale with the name nano-electro-mechanical systems (NEMS). These devices allow the study and detection of objects at the molecular scale [6,7] and also at the quantum scale [8]. The constant times are likewise reduced which implies a limited response time only because of the electronics control and not the NEMS itself. In addition, in the nanoscale era, we can see a modification in the intrinsic properties of materials as in silicon nanowires and their thermal and conductive properties modified by the size effect. Hearing implants are technological devices developed to correct hearing loss. Today, the cochlear implant is the most complete system. It implements the fields of acoustics, electronics, signal processing and information, biology, and knowledge of the human physiology. The objective of the microphone is to transduce acoustic waves to an electrical signal. Consequently, it operates in the frequency range from $20 \mathrm{~Hz}$ to $20 \mathrm{kHz}$ [9].

The objective of this paper is to implement a nano- and micro-electro-mechanical system (M\&NEMS) microphone with a low-noise instrumentation amplifier, which has a high signal-to-noise ratio (SNR) and high unity-gain bandwidth (UGBW). The architecture of the amplifier is carefully selected and some circuit innovations are explored. In addition, a detailed noise analysis of the complete system composed by the M\&NEMS microphone and the low-noise amplifier is presented. The complete system is fabricated in $65 \mathrm{~nm}$ CMOS process and measurement results are discussed. The rest of the paper is organized as follows. Section 2 presents an overview of our fabricated M\&NEMS microphone. Then, Section 3 provides the instrumentation amplifier details. Section 4 presents the measurement results of the fabricated circuit and discussion. Finally, Section 5 concludes the paper.

\section{Nano- and Micro-Electro-Mechanical System (M\&NEMS) Microphone Design}

The fundamental of M\&NEMS technology is presented for the first time in 2009 [10]. M\&NEMS technology allows designing a novel sensor that combines both MEMS and NEMS structures like the design of a 3D accelerometer sensor [10]. The M\&NEMS in-plane accelerometer is shown in Figure 1.

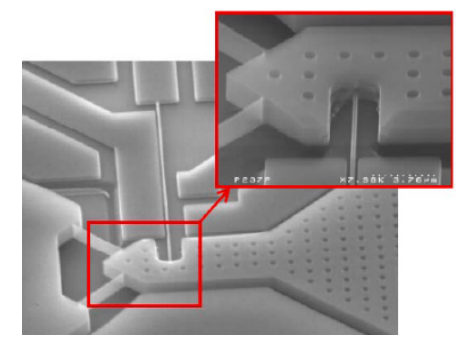

Figure 1. Scanning electron microscope (SEM) image of nano- and micro-electro-mechanical system (M\&NEMS) nanowire sensor [10].

Figure 2 shows the M\&NEMS transducer composed of four rigid micro-beams and four suspended silicon nanogauges. All micro-beams are placed between the inlet vents and the outlet vents. Inside the transducer, the inlet vents allows for guiding the sound wave and the outlet vents allows the equilibration of the pressure in a back cavity to be enabled. In addition, the motion of the beams is enabled by micro-slits placed between the beams and both top and bottom wafers.

Therefore, the pressure can easily drop from one side to the other side in the same beam. Inside, the suspended silicon gauges is located the stress that induced by the motion of a beam. It represents a transducer with a resistance variation of each silicon nanogauge. To measure the resistance variation, the four silicon nanogauges are connected as a Wheatstone bridge. If a sound pressure is detected, 
then two silicon nanogauges will be stretched and two silicon nanogauges will be compressed at the same time. Moreover, random accelerations are automatically self-canceled.

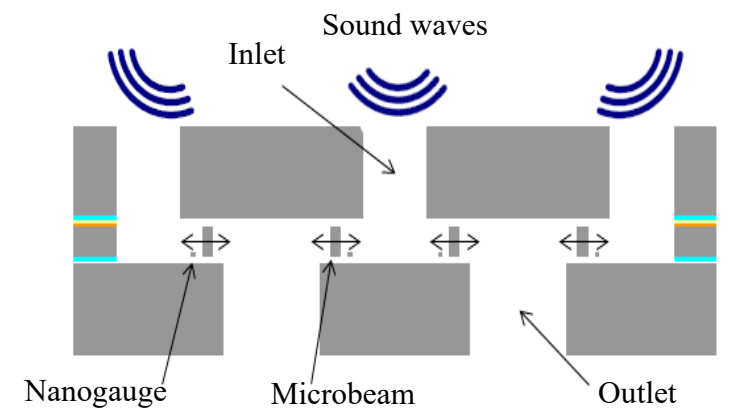

Figure 2. Microphone cross-section with sensing elements and acoustic configuration [11].

The technological M\&NEMS fabrication process is done in the clean room of the CEA-LETI (Atomic Energy Commission-Laboratory of Electronics Information Technology). Figure 3 shows the fabricated M\&NEMS accelerometer with zooming on different components like the four silicon nanogauges, the inlet vents and the outlet vents. The detailed analysis of the sensor's operation allows identifying the constraints dedicated to the bridge itself. Among these constraints, there is the common-mode voltage and the output impedance, as well as the constraints related to the audio application.

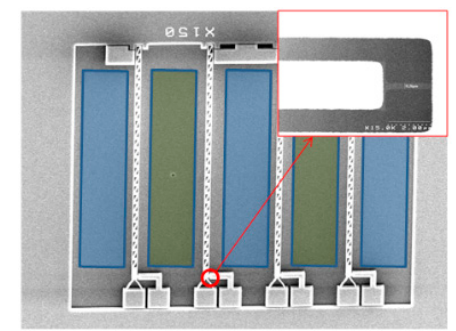

Figure 3. Microphone top view with the nanogauge and the micro-beams [12].

The M\&NEMS analog front-end composed by a sensor biasing circuit and an instrumentation amplifier as a read-out circuit is shown in Figure 4. The Wheatstone bridge is composed by the nanowire gauges. It is biased by a voltage-controlled constant current source. Moreover, the common mode voltage of the instrumentation amplifier input is maintained at $V_{\mathrm{dd}} / 2$ with a servo-loop build around A1 that equate source and sink current through the bridge. The differential voltage $V_{\text {in }}$ flowing through the instrumentation amplifier is proportional to the gauge resistance imbalance induced by acoustic vibrations. The power consumption of the electronic circuit is fixed by the current biasing. In the simplest case, the same power supply can bias both sensor and amplifier. In this case, the equivalent resistance $R_{E}$ of the four gages is quite high to maintain a low-power consumption. Moreover, the supply voltage of the complete M\&NEMS nanowire sensor is sufficiently small. However, all current integrated circuits in the industry generally operate with supply voltages greater than 1.2-V. This minimum supply voltage of the M\&NEMS nanowire sensor generates a bias $\mathrm{I}_{\mathrm{bias}}$ of about $279-\mu \mathrm{A}$. This bias current corresponds to a power supply of about $335-\mu \mathrm{W}$, which is excessive, on the one hand, because the nanowires cannot dissipate it, and on the other hand, it leads to an increase of the total power consumption that is not compatible with the target IoT application. Therefore, it is necessary to add a circuit that controls the voltage bias of the sensor independently of the amplifier supply voltage. The sensor output common-mode voltage might cause problems for the amplifier when the circuit is referenced at $0 \mathrm{~V}$. In fact, for a $100 \mu \mathrm{A} \mathrm{I}_{\text {bias }}$ current, the bias voltage $\mathrm{V}_{\text {bias }}$ is $0.4 \mathrm{~V}$. The sensor common-mode voltage $\mathrm{V}_{\mathrm{CM}}$ is about $0.2 \mathrm{~V}$ in this case. This $\mathrm{V}_{\mathrm{CM}}$ value is assuming the same gauges. Therefore, the amplifier input signals $\mathrm{V}_{\mathrm{A}}$ and $\mathrm{V}_{\mathrm{B}}$ evolve around an average value of $0.2 \mathrm{~V}$. With the objective of driving this $\mathrm{V}_{\mathrm{CM}}$ voltage to a value compatible with that of the amplifier 
without generating an excessive bias current, it is necessary to control the current of the $\mathrm{V}_{\mathrm{CM}}$ voltage independently, and therefore to control $\mathrm{V}_{\text {bias }}$ as shown in Figure 4.

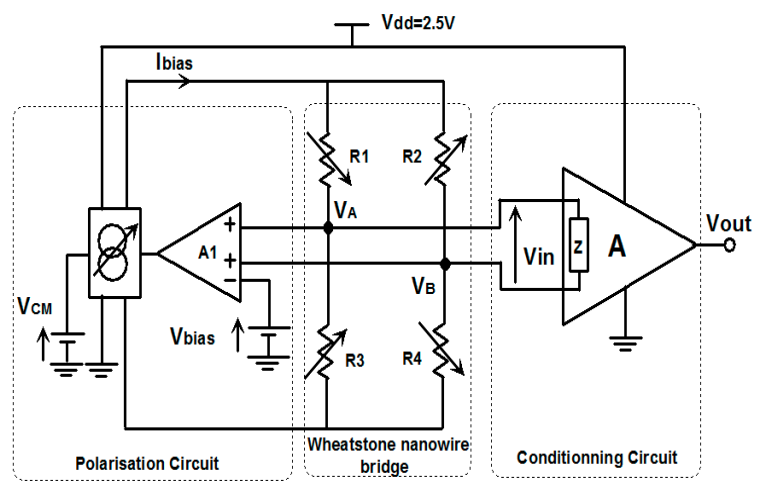

Figure 4. M\&NEMS nanowire sensor analog front-end.

The voltage $V_{\text {bias }}$ is one of the key parameters of sensitivity as expressed in Equation (1). It can also be considered that at constant sound pressure, the sensitivity affects the power of the output signal. If the $\mathrm{V}_{\text {bias }}$ increases, the output voltage also increases. Therefore, the polarization affects the SNR. However, the current flow through the sensor implies power consumption, which is a crucial parameter in battery-powered systems. Therefore, to optimize the sensor polarization, it is relevant to evaluate its SNR according to its power consumption. The impact of the bias voltage on the SNR is evaluated with the noise model. The thermomechanical noise occurs upstream of the bridge. From the models, the power $\mathrm{P}_{\text {signal }}$ of the useful signal is related to the voltage $\mathrm{V}_{\text {bias }}$ as:

$$
\mathrm{P}_{\text {signal }}=\left[\mathrm{V}_{\text {bias }} \frac{\Delta \mathrm{R}}{\mathrm{R}}\right]^{2} \text {. }
$$

According to the common definition of the SNR with a reference sound pressure of $94 \mathrm{~dB}-\mathrm{SPL}$, the SNR of the microphone alone is obtained with the following expression:

$$
\mathrm{SNR}=\frac{\left[\mathrm{V}_{\text {bias }} \frac{\Delta R}{R}\right]^{2}}{\int_{\mathrm{f}_{1}}^{\mathrm{f}_{2}} \mathrm{~V}_{\text {Total }}^{2}},
$$

where $\mathrm{V}^{2}$ Total denotes the total output noise power of the sensor. Therefore, the power consumption $\mathrm{P}_{\mathrm{absorb}}$ depends on the nominal value $\mathrm{R}_{0}$ of one nanogauge and can be written as:

$$
\mathrm{P}_{\mathrm{absorb}}=\frac{\mathrm{V}_{\text {bias }}^{2}}{\mathrm{R}_{0}} .
$$

These relations allow us to draw the curve of Figure 5, which shows the SNR evolution according to the supply power absorbed by the bridge. The signal-to-noise ratio reaches its asymptote (SNR Max of the sensor alone $65 \mathrm{~dB}$ ) when it is polarized with $2 \mathrm{~V}$ supply. The operating point of the sensor alone is considered optimal when the SNR stops to increase linearly with the power, from $43 \mu \mathrm{W}$.

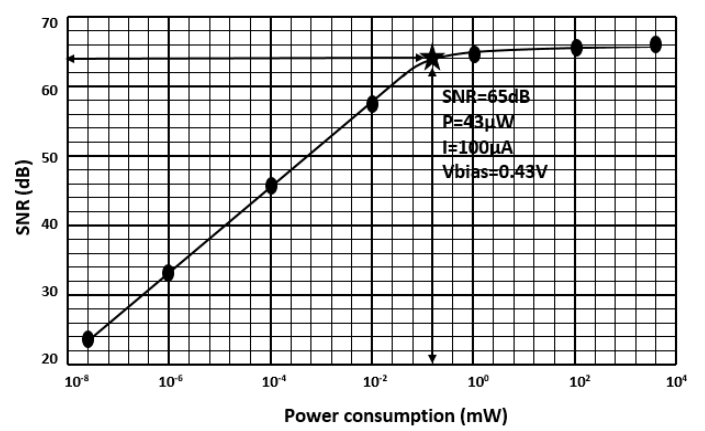

Figure 5. Signal-to-noise ratio (SNR) of the M\&NEMS nanowire sensor. 


\section{Low-Noise Instrumentation Amplifier Implementation}

Integrated circuits dedicated to measuring the resistance of silicon nanogauge in audio applications are not commercially available. Based on circuits already made for similar sensors where a Wheatstone bridge is used, a special instrument was developed. It allows the sensor to operate, demonstrates the relevance of the resistive detection, and studies its performance. Some results can also be used for the design of an integrated circuit. Consequently, the dedicated electronics should allow operating the sensor, to reach these maximum performances and to provide an easily exploitable signal. The integrated circuit must also, and above all, ensure the polarization of the bridge. According to the experimental development step of the nanogauges, the integrated device must propose a regulation of the current and several configurations. Protection functions have been added to preserve the gauges during manipulations. Its use must be easy to multiply the experiments, without the risk of damaging neither a sensor nor the instrument.

Flowing a current through the sensor elements is essential to obtain a signal. The easiest way to do this is to use the feed directly as shown in Figure 6. The use of a servo-control allows maintaining the current biasing independently of both the supply voltage and the value of the nanowire resistors. It also keeps the common mode close to the optimal value required for the proper operation of the amplification stage. The control loop comprises an adder that extracts the common-mode voltage of the bridge, a subtractor $\mathrm{S} 1$, an integrator I1, and a generator controlling the voltage $\mathrm{V}_{\mathrm{N}}$. The voltage $\mathrm{V}_{\text {error }}$ is the error voltage that can be written as:

$$
\mathrm{V}_{\text {error }}=\mathrm{V}_{\mathrm{REF}}-\mathrm{V}_{\mathrm{CM}} \text {. }
$$

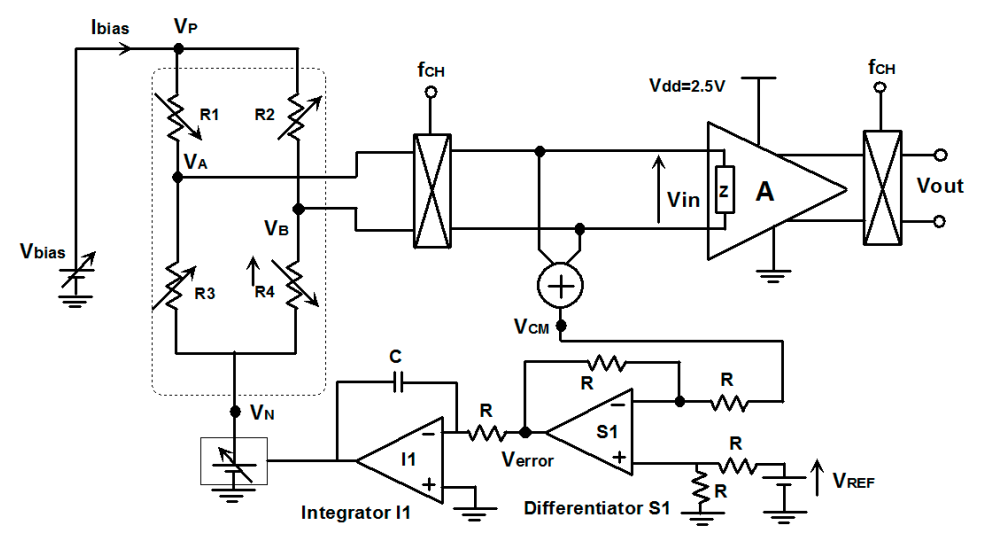

Figure 6. Voltage biasing of the M\&NEMS nanowire sensor.

At equilibrium, $\mathrm{V}_{\text {error }}=0 \mathrm{~V}$, then:

$$
\mathrm{V}_{\mathrm{N}}=\mathrm{V}_{\mathrm{CM}}-\frac{\mathrm{V}_{\text {bias }}}{2}
$$

Thus, the voltage $\mathrm{V}^{\prime}$ bias across the nanogauges bridge is controlled via the voltage $\mathrm{V}_{\text {bias }}$ such that:

$$
\mathrm{V}_{\text {bias }}^{\prime}=\frac{\mathrm{V}_{\text {bias }}}{2} \text {. }
$$

This method can also be applied to the current polarization [9]. The diagram is given in Figure 6. The control loop composed by the subtractor $\mathrm{S} 1$ followed by the integrator I1 acts on the current $\mathrm{I}_{\mathrm{N}}$ to cancel the common residual mode voltage $\mathrm{V}_{\mathrm{CM}}$ generated at the input of the amplifier when there is an imbalance between $I_{P}$ and $I_{N}$. As a result, at equilibrium, the currents $I_{P}$ and $I_{N}$ are identical. The accuracy required depends on the common-mode tolerance of the amplifier.

This configuration is easily achievable in CMOS technology because a transistor is naturally a voltage-controlled current source. It has the advantage of completely dissociating the useful bias 
current from the undesired common-mode current. It is enough to control $\mathrm{I}_{\text {bias }}$ to master the current that crosses the sensor, and thus its sensitivity. $\mathrm{I}_{\text {bias }}$ is a lever for acting on sensitivity.

The two-stage instrumentation amplifier structure has the high input impedance required to maintain the sensitivity of the resistive sensor [13]. A low-noise instrumentation amplifier is integrated after the Wheatstone bridge. It processes a voltage signal $V_{A}-V_{B}$. It is composed of amplifiers $A 1, A 2$ and $\mathrm{A} 3$ as shown in Figure 7. The architecture of each amplifier is based on a two-stage operational transconductance amplifier (OTA) as shown in Figure 8.

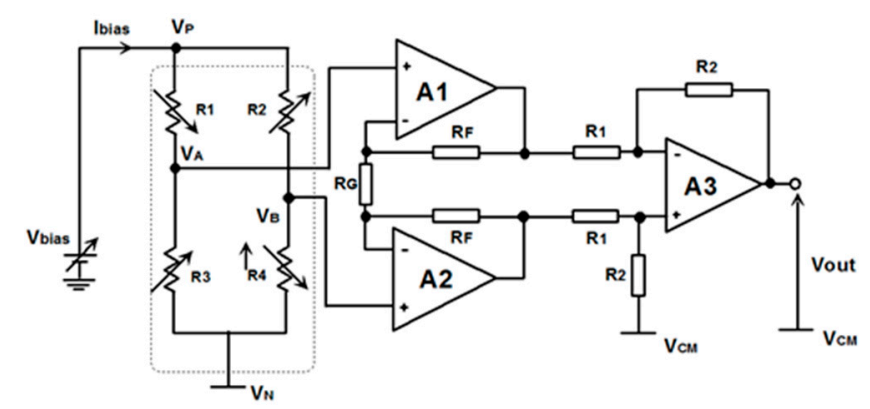

Figure 7. Instrumentation amplifier.

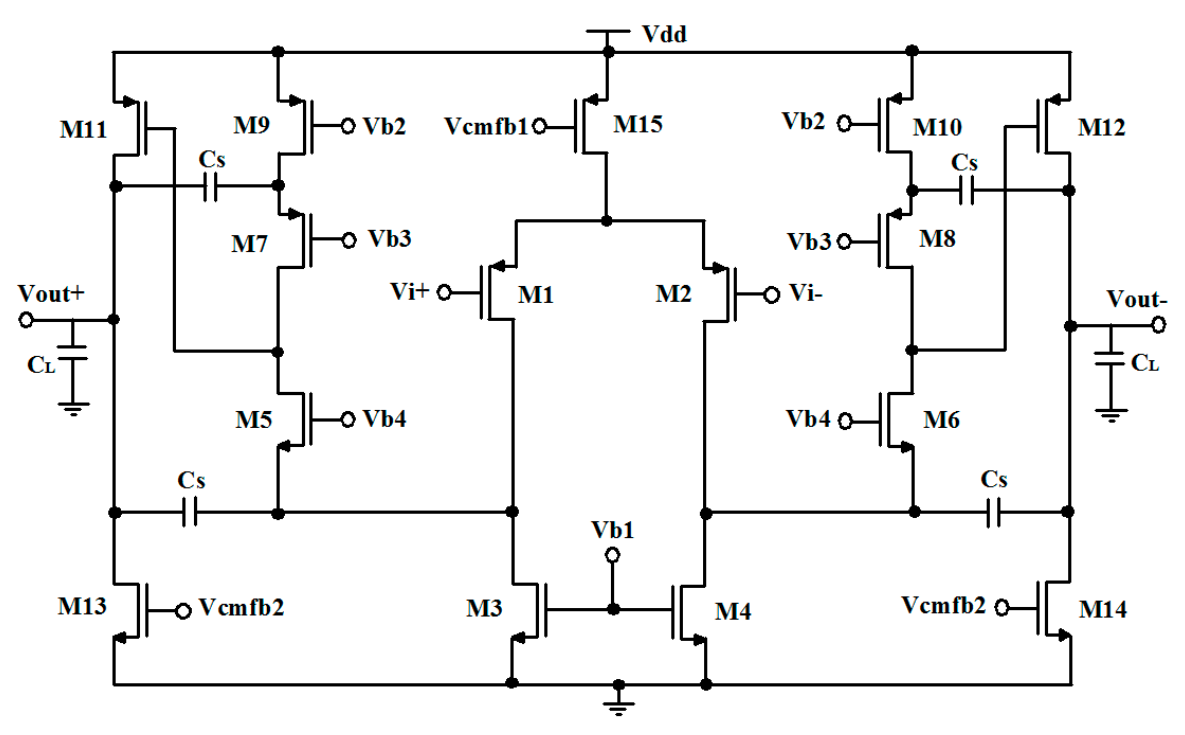

Figure 8. Two-stage operational transconductance amplifier (OTA).

The input stage is directly connected to the sensor. It allows amplifying the difference voltage $\mathrm{V}_{\mathrm{A}}-\mathrm{V}_{\mathrm{B}}$. It is composed of amplifiers $\mathrm{A} 1$ and $\mathrm{A} 2$ whose intrinsic gain is very high. The associated passive elements $R_{F}$ and $R_{G}$ determine the voltage gain $G_{V 1}$ of the entire stage such that:

$$
\mathrm{G}_{\mathrm{V} 1}=1+\frac{2 \mathrm{R}_{\mathrm{F}}}{\mathrm{R}_{\mathrm{G}}}
$$

The second amplification stage can convert the differential signal into a voltage $\mathrm{V}_{\mathrm{s}}$, referenced to the common-mode voltage. Its gain $G_{V 2}$ depends on the elements $R_{2}$ and $R_{1}$ such that:

$$
\mathrm{G}_{\mathrm{V} 2}=\frac{\mathrm{R}_{2}}{\mathrm{R}_{1}} .
$$

The total gain $\mathrm{G}_{\mathrm{V}}$ of the instrumentation amplifier can be written as:

$$
\mathrm{G}_{\mathrm{V}}=\mathrm{G}_{\mathrm{V} 1} \times \mathrm{G}_{\mathrm{V} 2}=\frac{\mathrm{R}_{2}}{\mathrm{R}_{1}}\left(1+\frac{2 \mathrm{R}_{\mathrm{F}}}{\mathrm{R}_{\mathrm{G}}}\right) .
$$


The resistors' network of the instrumentation amplifier was designed by $R_{1}=5.1 \mathrm{k} \Omega, R_{2}=160 \mathrm{k} \Omega$, $R_{F}=2 \mathrm{k} \Omega$ and $R_{G}=390 \Omega$. Therefore, the ideal total differential gain $G_{V}$ is $51 \mathrm{~dB}$. Finally, the sensitivity of the sensor is increased by a factor $\mathrm{k}$ comprising the product of the gains of the stages of the amplification chain. This simple structure involves the bias voltages $V_{P}$ and $V_{N}$ across the sensor. They define both the current $\mathrm{I}_{\text {bias }}$ flowing through it and the common-mode voltage $\mathrm{V}_{\mathrm{CM}}$ according to Equations (10) and (11) as:

$$
\begin{gathered}
I_{\text {bias }}=\frac{V_{P}-V_{N}}{R_{0 \text { eq }}}, \\
V_{C M}=\frac{V_{P}-V_{N}}{2}\left[\frac{R_{3}}{R_{1}+R_{3}}+\frac{R_{4}}{R_{2}+R_{4}}\right],
\end{gathered}
$$

where $R_{0 \text { eq }}$ is the equivalent resistance of the sensor and $R_{n}$ is the resistance of element $n$. The common-mode voltage $\mathrm{V}_{\mathrm{CM}}$ can be critical for the proper functioning of the sensor and its electronics. It must be located between the supply voltage of the amplifier and the reference voltage to allow the excursion of the output voltages of the sensor without saturating the amplifiers that make up the chain. The value $\mathrm{V}_{\mathrm{DD}} / 2$ allows the maximum excursion. Therefore, this choice implies conditions on the voltages $V_{P}$ and $V_{N}$. In the case of a sensor preceded by this amplification, structure supplied with a voltage of $2.5 \mathrm{~V}$, for a polarization current $\mathrm{I}_{\text {bias }}$ of $100 \mu \mathrm{A}$, with nominal nanogauge resistance $\mathrm{R}_{0}$ of $4300-\Omega$, the voltage $\mathrm{V}_{\mathrm{P}}$ must be $1.365 \mathrm{~V}, \mathrm{~V}_{\mathrm{CM}}$ of $1.25 \mathrm{~V}$ and $\mathrm{V}_{\mathrm{N}}$ of $1.135 \mathrm{~V}$.

The bandwidth of the instrumentation amplifier is a significant limiting factor. It must be adapted to the sensor and its application [14]. Quantities that change slowly as the temperature or the acceleration does not require the same bandwidth as fast variables as the acoustic vibrations of air. In an instrumentation amplifier, the resulting bandwidth is expressed as a function of the gain-bandwidth product of the operational amplifiers. It will be necessary to find a satisfactory compromise between the gain and the bandwidth by considering the intrinsic limits of the operational amplifiers. Therefore, it is according to one of these conditions that the distribution of the gains in an amplification chain is determined.

To reduce the input-equivalent noise of the instrumentation amplifier, we propose to use the well-known chopper stabilization (CHS) technique. The traditional CHS technique is shown in Figure $9[15,16]$. The signal path mismatch and the demodulated current spikes generate a residual offset $\mathrm{V}_{\text {os }}$. Therefore, alternating current (AC) spike is caused by the mismatch between the capacitances due to clock feed-through at the Chopper clocks transition moments. The first modulator M1 rectify this AC current. Therefore, a DC spike current appears at its input. The resulting DC spike current has an average value $\mathrm{I}_{\text {offset }}$ of:

$$
\mathrm{I}_{\text {offset }}=2\left(\Delta \mathrm{C}_{1}-\Delta \mathrm{C}_{2}\right) \mathrm{V}_{\mathrm{clk}} \mathrm{f}_{\mathrm{CH}},
$$

where $\Delta C_{1}$ and $\Delta C_{2}$ denote the CHS mismatch parasitic capacitance, $V_{\text {clk }}$ denotes the clock signal magnitude and $\mathrm{f}_{\mathrm{CH}}$ denotes the chopping clock frequency, with $\mathrm{f}_{\mathrm{CH}}=12-\mathrm{kHz}$. The chopper series impedance and the input signal source are going through by this noise current. Therefore, it depicts as an input voltage spike. The residual offset $\mathrm{V}_{\mathrm{os}}$ resulting from the spike average $\mathrm{DC}$ value can be written as:

$$
\mathrm{V}_{\text {os }}=2 \mathrm{R}\left(\Delta \mathrm{C}_{1}-\Delta \mathrm{C}_{2}\right) \mathrm{V}_{\mathrm{clk}} \mathrm{f}_{\mathrm{CH}},
$$

where $\mathrm{R}$ denotes the equivalent input impedance. Therefore, a residual offset $\mathrm{V}_{\text {os }}$ depicts the spike average DC value. Moreover, a spike voltage $\mathrm{V}_{\text {os }}$ is created in the input of Modulator1. This spike voltage causes a low-frequency interference. To cancel-out this interference, the solution is to create a proper delay $\Delta t$ between Modulator1 and Modulator2. The proposed instrumentation amplifier CHS technique is shown in Figures 10 and 11. The instrumentation amplifier with its common-mode feedback circuit (CMFB) is located between two modulating clock signals $\mathrm{m} 1(\mathrm{t})$ and $\mathrm{m} 2(\mathrm{t})$ with period T. Moreover, we introduce a delay $\Delta \mathrm{t}$ between the two clock signals $\mathrm{m} 1(\mathrm{t})$ and $\mathrm{m} 2(\mathrm{t})$ at the same time. Due to the introduction of the delay $\Delta t$, this technique causes a chopping of the spike signal itself. Therefore, the DC content of the output signal $\mathrm{V}_{\text {out }}(\mathrm{t})$ is minimized. The residual output dc offset is completely cancelled if an optimal delay value $\Delta \mathrm{t}_{\mathrm{opt}}$ exists, which can be written as: 


$$
\Delta \mathrm{t}_{\mathrm{opt}}=\ln (2) \times \tau,
$$

where $\tau=R \times C_{i n}$ with $R$ denotes the input resistance and $C_{i n}$ denotes the input capacitance of the amplifier. The major weakness of this technique is the $\tau$ itself, which not only depends on the sensor's source resistance $R$, but also on the amplifier's input capacitance $C_{i n}$.

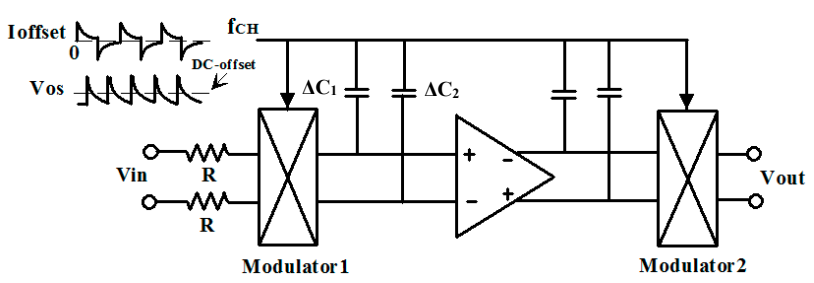

Figure 9. Chopper stabilization clock feed-through circuit.

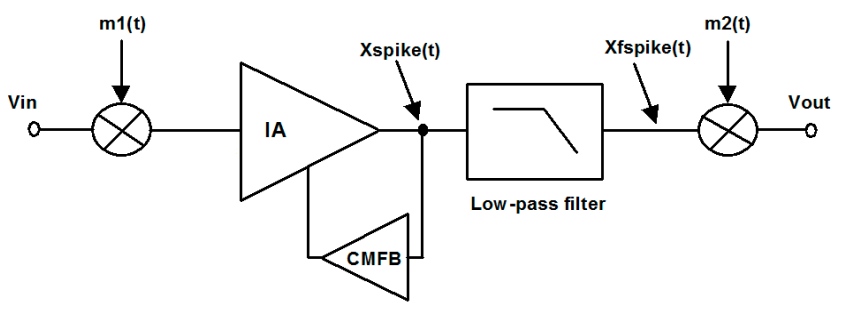

Figure 10. Circuit diagram of the delay chopper stabilization with spike shaping.
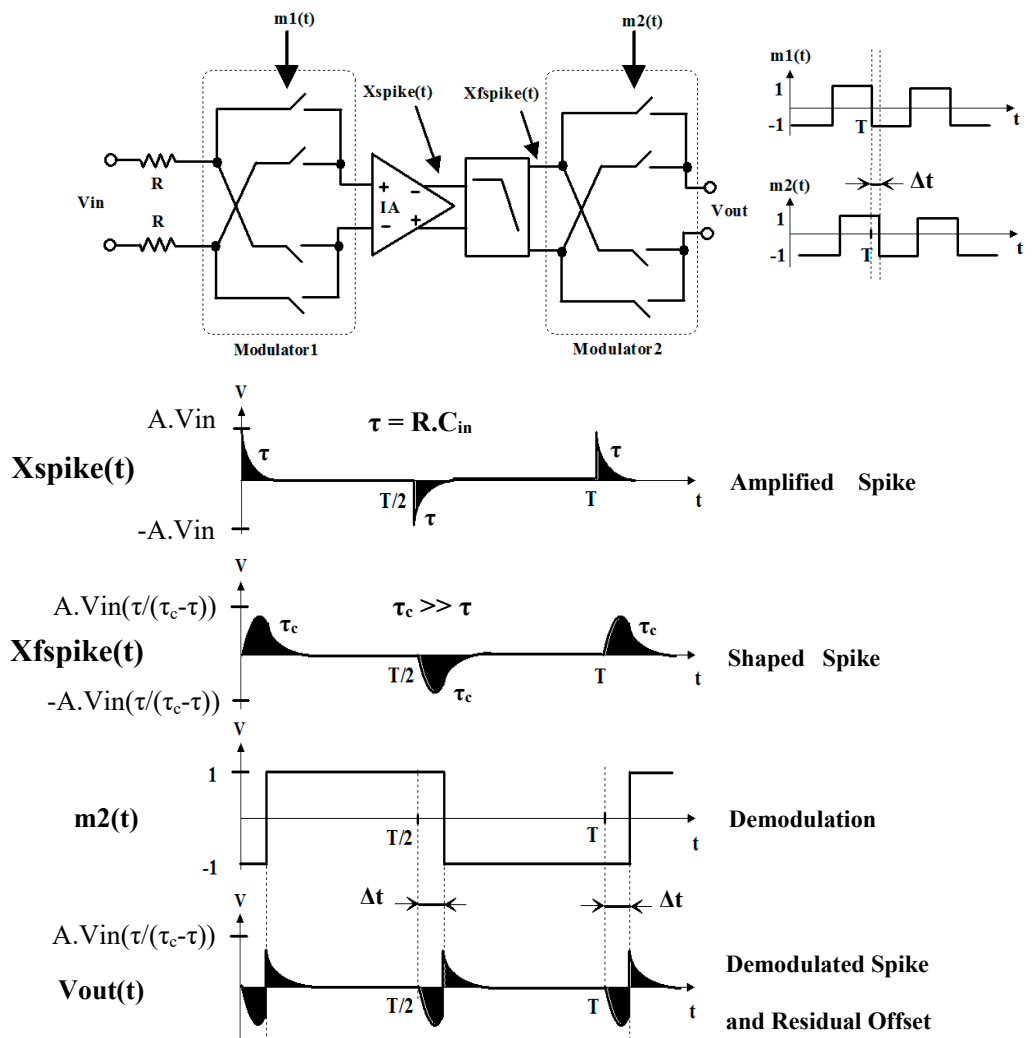

Figure 11. Delayed chopper stabilization with spike shaping analysis.

The input modulator's spike signal is amplified and then multiplied with $\mathrm{m} 2(\mathrm{t})$ in the demodulator. The resulting output signal $V_{\text {out }}(t)$ then contains, apart from higher order harmonics of the chopping frequency, a DC part or residual offset, which is due to chopping artifacts. To solve this problem, shaping of the spike can be introduced by the addition of a first order low-pass filter with time constant 
$\tau_{c}$ after the amplifier. We must have $T>>\tau_{c}>>\tau$ with $T$ is the period of the square wave signal $m 1(t)$. The shape of the time response of the filtered spike is primarily determined by $\tau_{c}$ and independent of the impedance of the connected sensor.

Since the output offset is still linearly dependent on $\tau$, the optimization of $\Delta t_{\text {opt }}$ has been done in such a way that offset reduction is most effective for a worst-case sensor resistance. For our specific implementation $\Delta \mathrm{t}_{\mathrm{opt}} / \tau_{\mathrm{c}}=0.8$ has been chosen. The low-pass filter has a cut-off frequency of $30 \mathrm{kHz}$. The nominal chopping frequency is $12 \mathrm{kHz}$.

\section{Measurement Results}

Prototypes of the M\&NEMS microphone with the low-noise instrumentation amplifier are fabricated and experimentally characterized. The die microphotograph with the layout view of the instrumentation amplifier is shown in Figure 12. The method of simultaneous comparison is used to perform the measurement of the M\&NEMS microphone, as shown in Figure 13. Some measurement devices are used to perform the test of the chip. A calibrated microphone is used as a reference microphone. It is placed near the tested microphone in the objective to compare their outputs. To isolate unwanted external phenomena, an anechoic chamber is used to perform all measurements. A loudspeaker is placed at a distance of one meter in front of the two microphones. The loudspeaker generates a sound with $1 \mathrm{kHz}$ frequency. It is used as a reference point. In the objective to obtain an equal response of loudspeaker at all frequencies, a corrected EQ (equalizer) curve is used. The output of the M\&NEMS microphone, as well as the output of the B\&K Type 2250 Analyzer (Brüel \& Kjær, Nærum, Denmark), are connected to the AP 2700 audio analyzer.

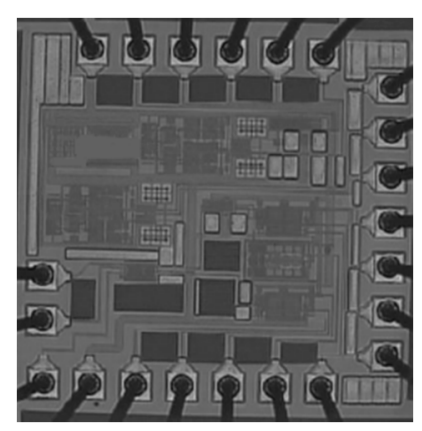

Figure 12. Chip microphotograph of the instrumentation amplifier in CMOS 65-nm technology and $1 \mathrm{~mm}^{2}$ area.

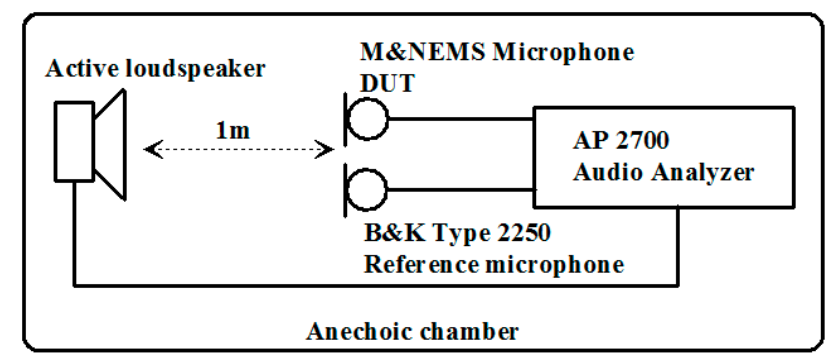

Figure 13. Measurement setup of the hybrid circuit composed by the M\&NEMS microphone and the $\Delta \Sigma$ modulator.

The sound pressure of the loudspeaker is fixed at $94 \mathrm{~dB}$ with a frequency of $1 \mathrm{kHz}$. The B\&K analyzer detects the sound pressure level. The $94 \mathrm{~dB}$ level corresponds to a $1 \mathrm{~Pa}$ acoustic sound pressure level. To obtain a constant level, the frequency response is corrected by the EQ curve. Therefore, the reference microphone frequency is fixed at $1 \mathrm{kHz}$. The comparison of the level of all other frequencies to the reference frequency is shown in Figure 14. The sound pressure level of $94 \mathrm{~dB}$ 
corresponds to the normalized response of $0 \mathrm{~dB}$. It is clear from Figure 14, the M\&NEMS microphone has a flat analog output response to about $20 \mathrm{kHz}$. After performing the frequency response of the M\&NEMS microphone, the measurement of the SNR of the instrumentation amplifier was performed and described below.

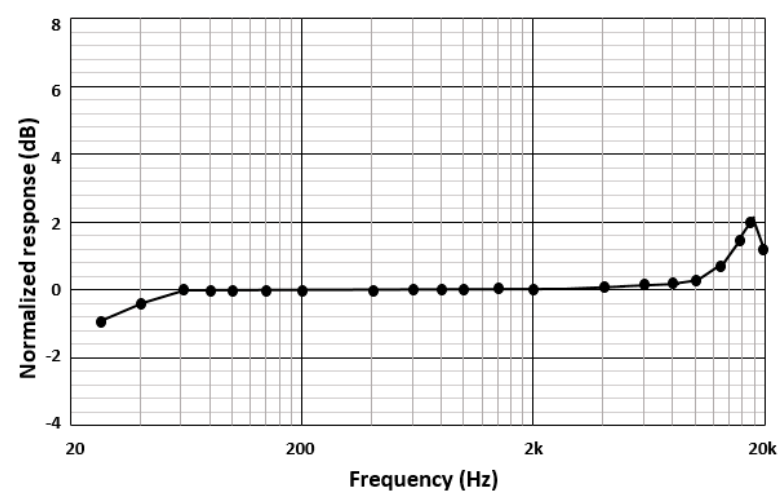

Figure 14. M\&NEMS microphone output versus frequency at sound source level of $94 \mathrm{~dB}$ in the $20 \mathrm{~Hz}-20 \mathrm{kHz}$ frequency range.

In a first step, to measure the instrumentation amplifier, no signal coming from the M\&NEMS microphone is injected at its inputs. The ammeter inserted in the supply circuit to measure the current $I_{\text {Supply }}$ consumed by this part of the circuit as a function of the voltage $V_{\text {biasAI }}$. The measurement results allow us to know the relationship between the current absorbed by the instrumentation amplifier as a function of the bias voltage $V_{\text {bias }}$. The curve plotted with experimental data and those obtained with the results of simulations for different models of transistors are shown in Figure 15 for comparison. Simulation results are performed in three processes and temperature corners as TT for Typical-Typical, FF for Fast-Fast and SS for Slow-Slow. The curve observed experimentally is similar to that obtained in the simulation with the SF-transistor model. Therefore, the deviation observed when the current exceeds $160 \mu \mathrm{A}$ can be attributed to the presence of an underestimated access resistance during the simulation. This first characterization identifies the transistor model closest to that of the transistors integrated into the chip.

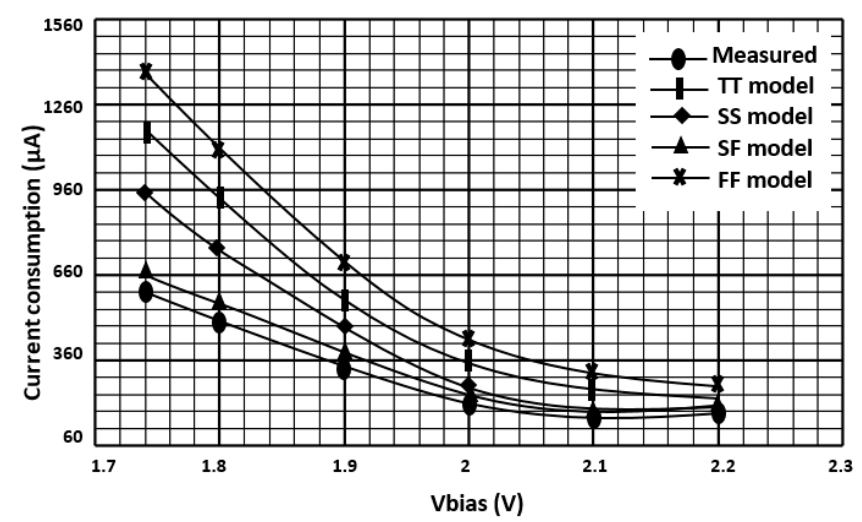

Figure 15. Direct current (DC) consumption of the instrumentation amplifier with bias voltage.

The curves in Figure 16 show the SNR of the complete system as a function of the amplifier power consumption. Simulation results have been added for comparison. An essential parameter to evaluate the proper function of the low-noise instrumentation amplifier is its input-referred noise. From Figure 17, the measured input-referred noise of the instrumentation amplifier is about $12 \mathrm{nV} / \sqrt{ } \mathrm{Hz}$. It appears that the evolution of the experimentally measured SNR is consistent with that obtained with the simulation models. The maximum measured SNR is $77 \mathrm{~dB}$. This value is nearly the same as the 
simulation result. It is obtained for a current of $160 \mu \mathrm{A}$. It corresponds to a power consumption of $0.4 \mathrm{~mW}$. Therefore, this power consumption is still excessive for the complete circuit.

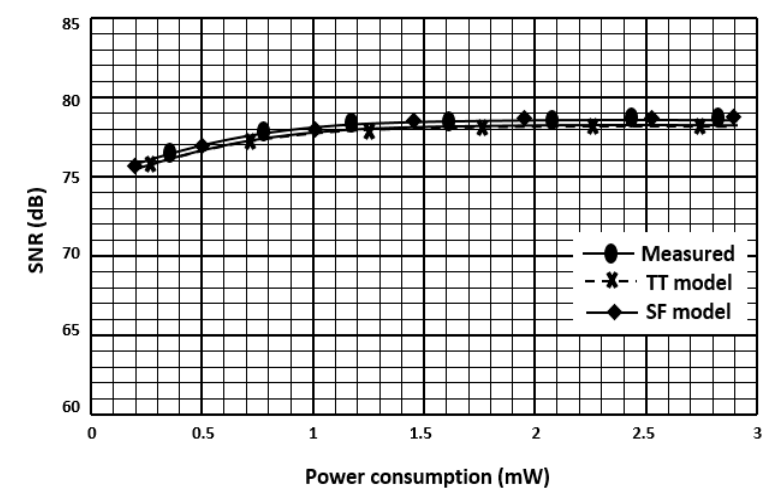

Figure 16. SNR of the instrumentation amplifier versus power consumption.

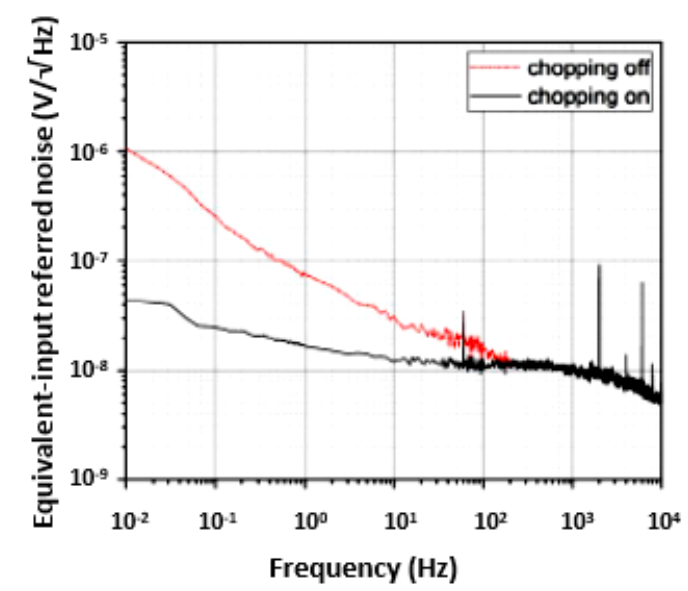

Figure 17. Instrumentation amplifier equivalent-input referred noise.

The measurement results of our low-noise instrumentation amplifier and the comparison with other instrumentation amplifiers is reported in Table 1. A fundamental parameter is used to evaluate the overall power efficiency of the instrumentation amplifier. This parameter is the noise efficiency parameter (NEF), which can be written as [17]:

$$
\mathrm{NEF}=\mathrm{V}_{\mathrm{rms}} \sqrt{\frac{2 \cdot \mathrm{I}_{\text {total }}}{\pi \cdot \mathrm{U}_{\mathrm{th}} \cdot 4 \mathrm{kT} \cdot \mathrm{BW}}}
$$

where $\mathrm{I}_{\text {total }}$ is the total current consumption, $\mathrm{V}_{\text {rms }}$ is the RMS (root-mean-square) input-referred noise, $\mathrm{U}_{\mathrm{th}}$ is the thermal voltage and BW is the bandwidth of the instrumentation amplifier. From Equation (15), it is clear that the NEF parameter includes almost every performance shown in Table 1, namely the equivalent-input referred noise, the power consumption, the bandwidth and indirectly the PSRR (power-supply rejection ratio) and the CMRR (common-mode rejection ratio). In [18,19], the instrumentation amplifier has a high CMRR and high PSRR. However, it has also a high equivalent-input referred noise at list of about $18 \mathrm{nV} / \sqrt{ } \mathrm{Hz}$. In [20-22], the measured instrumentation amplifier has a low CMRR and low PSRR. Moreover, it has a worse equivalent-input referred noise at least $36 \mathrm{nV} / \sqrt{\mathrm{Hz}}$. Therefore, this noise level affects drastically the instrumentation amplifier and degrades its performances. As a result, all compared instrumentation amplifiers have an equivalent-input referred noise greater than $18 \mathrm{nV} / \sqrt{ } \mathrm{Hz}$. On the other hand, our measured instrumentation amplifier has a high CMRR and high PSRR. Moreover, it has the lowest equivalent-input referred noise of only $12 \mathrm{nV} / \sqrt{ } \mathrm{vHz}$. As a result, for the same performances, our instrumentation amplifier has a good tradeoff between the 
supply voltage, the PSRR and the CMRR. Our circuit achieves a NEF of 3.7, a PSRR of $108 \mathrm{~dB}$ and a CMRR of $121 \mathrm{~dB}$. Therefore, it proves a competitive performance compared to the state-of-the-art.

Table 1. Performances comparison of the instrumentation amplifier with the state-of-the art.

\begin{tabular}{ccccccc}
\hline Specifications & [18] & [19] & [20] & [21] & [22] & This Work \\
\hline Technology, nm & 180 & 320 & 130 & 40 & 180 & 65 \\
Supply, V & 3.3 & 3.3 & 1.2 & 1.2 & 0.8 & 2.5 \\
Power, $\mu \mathrm{W}$ & 558 & 561 & 3 & 2 & 8 & 400 \\
CMRR, dB & 162 & 120 & 85 & 87 & 85 & 121 \\
PSRR, dB & 111 & 115 & - & - & 74 & 108 \\
Bandwidth, Hz & $59 \mathrm{k}$ & $40 \mathrm{k}$ & $5 \mathrm{k}$ & $20 \mathrm{k}$ & 670 & $20 \mathrm{k}$ \\
Noise, $\mathrm{nV} / \sqrt{ } \mathrm{Hz}$ & 28.3 & 18 & 47 & 110 & 36 & 12 \\
NEF & 4.2 & 10.6 & 3.9 & 4.9 & 2.1 & 3.7 \\
\hline
\end{tabular}

\section{Conclusions}

In this paper, a low-noise low power and highly sensitive M\&NEMS microphone for the use in IoT is presented. The resistive accelerometer and the electronic interface are, respectively, silicon nanowires and a low-noise instrumentation amplifier. Several low-noise and low-power techniques are developed at circuit and system levels in order to decrease both the power consumption and the noise and maintain a good performance. Because the most critical noisy block is the amplifier, the CHS technique is implemented around this block to eliminate its offset and $1 / \mathrm{f}$ noise. The instrumentation amplifier is implemented in a $65 \mathrm{~nm}$ CMOS technology. The supply voltage is $2.5 \mathrm{~V}$ while the power consumption is $0.4 \mathrm{~mW}$. The core area is $1 \mathrm{~mm}^{2}$. The hybrid circuit composed by the M\&NEMS microphone and the instrumentation amplifier was fabricated and measured. From measurement results over a signal bandwidth of $20 \mathrm{kHz}$, the low-noise instrumentation amplifier achieves an SNR of $77 \mathrm{~dB}$ and it has a great potential of being used in IoT applications.

Author Contributions: Conceptualization, J.N. and P.M.F.; methodology, J.N.; validation, J.N., P.M.F. and S.M.; formal analysis, S.M.; resources, J.N.; writing—original draft preparation, J.N.; writing—review and editing, J.N and P.M.F.; supervision, J.N. All authors have read and agreed to the published version of the manuscript.

Funding: This research received no external funding.

Acknowledgments: This work was conducted in cooperation with Prince Sattam bin Abdulaziz University, Alkharj, Saudi Arabia, and Sorbonne Université, Paris-Saclay, CentraleSupélec, CNRS, Lab. de Génie Electrique et Electronique de Paris, France.

Conflicts of Interest: The authors declare no conflict of interest.

\section{References}

1. Barbour, N.; Hopkins, R.; Kourepenis, A.; Ward, P. Inertials MEMS systems and applications. Lect. NATO Sci. Technol. Organ. 2011, 116, 1-18.

2. Yazdi, N.; Ayazi, F.; Najafi, K. Micromachined inertial sensors. Proc. IEEE 1998, 86, 1640-1659. [CrossRef]

3. Bell, D.J.; Lu, T.J.; Fleck, N.A.; Spearing, S.M. MEMS actuators and sensors: Observation on their performance and selection for purpose. J. Micromech. Microeng. 2005, 15, 153-164. [CrossRef]

4. Duraffourg, L.; Laurent, L.; Moulet, J.; Arcamone, J.; Yon, J. Array of Resonant Electromechanical Nanosystems: A Technological Breakthrough for Uncooled Infrared Imaging. Micromachines 2018, 9, 401. [CrossRef] [PubMed]

5. Yole Developpement. Technology Trends for Inertial MEMS. 2012. Available online: https://www.yumpu.com/ en/document/read/9114254/technology-trends-for-inertial-mems-i-micronews (accessed on 20 March 2020).

6. Li, M.; Tang, H.X.; Roukes, M.L. Ultra-sensitive NEMS-based cantilevers for sensing, scanned probe and very high frequency applications. Nat. Nanotechnol. 2007, 2, 114-120. [CrossRef] [PubMed]

7. Naik, A.K.; Hanay, M.S.; Hiebert, W.K.; Feng, X.L.; Roukes, M.L. Towards single-molecule nanomechanical mass spectrometry. Nat. Nanotechnol. 2009, 4, 445-450. [CrossRef] [PubMed]

8. Keith, S.C.; Roukes, C.L. Putting mechanics into quantum mechanics. Phys. Today 2005, 58, 36-42. 
9. Kitchin, C.; Counts, L. A Designer's Guide to Instrumentation Amplifiers. 2006. Available online: https://www.analog.com/media/en/training-seminars/design-handbooks/designers-guide-instrumentamps-complete.pdf (accessed on 20 March 2020).

10. Robert, P.; Nguyen, V.; Hentz, S.; Duraffourg, L.; Jourdan, G.; Arcamone, J.; Harrisson, S. M\&NEMS: A new approach for ultra-low cost 3d inertial sensor. In Proceedings of the SENSORS, 2009 IEEE, Christchurch, New Zealand, 25-28 October 2009.

11. Nebhen, J.; Savary, E.; Rahajandraibe, W.; Dufaza, C.; Meillere, S.; Kussener, E.; Barthelemy, H.; Czarny, J.; Lhermet, H. Low-noise CMOS amplifier for readout electronic of resistive NEMS audio sensor. In Proceedings of the IEEE Symposium on Design, Test, Integration and Packaging of MEMS/MOEMS (DTIP), Cannes, France, 1-4 April 2014.

12. Savary, E.; Rahajandraibe, W.; Meillère, S.; Kussener, E.; Barthelemy, H.; Czarny, J.; Lhermet, H.; Robert, P. High resolution NEMS smart audio sensor based on resistive silicon nano wires for hearing aids. In Proceedings of the 21st IEEE International Conference on Electronics, Circuits and Systems (ICECS), Marseille, France, 7-10 December 2014; pp. 558-561.

13. Peyton, A.; Walsh, V. Analog Electronics with Op-Amps: A Source Book of Practical Circuits; Cambridge University Press: Cambridge, UK, 1993.

14. O'Grady, A. Transducer/sensor excitation and measurement techniques. Analog Dialogue 2000, 34, 1-6.

15. Yavari, M. Hybrid cascode compensation for two-stage CMOS op-amps. IEICE Trans. Electron. 2005, 88, 1161-1165. [CrossRef]

16. Blazquez, G.; Pons, P.; Boukabache, A. Capabilities and limits of silicon pressure sensors. Sens. Actuators 1989, 17, 387-403. [CrossRef]

17. Harrison, R.; Charles, C. A low-power low-noise CMOS amplifier for neural recording applications. IEEE J. Solid-State Circuits 2003, 38, 958-965. [CrossRef]

18. Kwon, Y.; Kim, H.; Kim, J.; Han, K.; You, D.; Heo, H.; Cho, D.; Ko, H. Fully Differential Chopper-Stabilized Multipath Current-Feedback Instrumentation Amplifier with R-2R DAC Offset Adjustment for Resistive Bridge Sensors. Appl. Sci. 2019, 10, 63. [CrossRef]

19. Butti, F.; Piotto, M.; Bruschi, P. A Chopper Instrumentation Amplifier with Input Resistance Boosting by Means of Synchronous Dynamic Element Matching. IEEE Trans. Circuits Syst. I Regul. Pap. 2017, 64, 753-794. [CrossRef]

20. Zheng, J.; Ki, W.H.; Hu, L.; Tsui, C.Y. Chopper Capacitively Coupled Instrumentation Amplifier Capable of Handling Large Electrode Offset for Biopotential Recordings. IEEE Trans. Circuits Syst. II Express Briefs 2017, 64, 1392-1396. [CrossRef]

21. Chandrakumar, H.; Markovic, D. A high dynamic-range neural recording chopper amplifier for simultaneous neural recording and stimulation. IEEE J. Solid-State Circuits 2017, 52, 645-656. [CrossRef]

22. Yaul, F.M.; Chandrakasan, A.P. A noise-efficient $36 \mathrm{nV} / \sqrt{ } \mathrm{Hz}$ chopper amplifier using an inverter-based 0.2-V supply input stage. IEEE J. Solid-State Circuits 2017, 52, 3032-3042. [CrossRef]

(C) 2020 by the authors. Licensee MDPI, Basel, Switzerland. This article is an open access article distributed under the terms and conditions of the Creative Commons Attribution (CC BY) license (http://creativecommons.org/licenses/by/4.0/). 